\title{
Experimental observation of the correlation between the interfacial Dzyaloshinskii-Moriya interaction and work function in metallic magnetic trilayers
}

\author{
Yong-Keun Park ${ }^{1,2}$, Dae-Yun Kim¹, Joo-Sung Kim, Yune-Seok Nam', Min-Ho Park', Hyeok-Cheol Choi', \\ Byoung-Chul Min $\mathbb{D}^{2}$ and Sug-Bong Choe ${ }^{1}$
}

\begin{abstract}
The Dzyaloshinskii-Moriya interaction (DMI) generates intriguing chiral magnetic objects, such as magnetic skyrmions and chiral domain walls, that can be used as building blocks in emerging magnetic nanodevices. Precise control of the DMI strength is one of the key issues for achieving better stability and functionality of these chiral objects. In this paper, we report that in magnetic trilayer films, the DMI strength exhibits a noticeable correlation with the work functions of the non-magnetic layers interfaced to the magnetic layer. This correlation with the intrinsic material parameters provides a guideline for material selection for engineering the DMI strength.
\end{abstract}

\section{Introduction}

Chiral magnetic materials, the phenomena associated with them, and the technological opportunities provided by emerging spintronic devices ${ }^{1,2}$ have recently attracted increasing academic attention. Such chiral magnetic phenomena are caused by an antisymmetric exchange interaction, which is the so-called Dzyaloshinskii-Moriya interaction (DMI). ${ }^{3,4}$ In magnetic thin films, a sizeable DMI generates built-in chirality of magnetic domain walls (DWs), which is essential for current-induced DW motion via spin-orbit torques (SOTs). ${ }^{5,6}$ The ultimate speed of the DW motion has been revealed to also be governed by the DMI strength. ${ }^{7}$ In addition, a sizeable DMI is an essential ingredient for generating topological objects, such as magnetic skyrmions, ${ }^{8,9}$ which can be used in high-density digital devices for racetrack memory and so on. ${ }^{1,2,8}$

Correspondence: Sug-Bong Choe (sugbong@snu.ac.kr) or

Byoung-Chul Min (min@kist.re.kr)

${ }^{1}$ Department of Physics and Institute of Applied Physics, Seoul National

University, Seoul 08826, Republic of Korea

${ }^{2}$ Center for Spintronics, Korea Institute of Science and Technology, Seoul

02792, Republic of Korea
Because of the academic interest and technological importance, numerous efforts have been devoted to understanding the DMI and its role on magnetic phenomena. Belabbes et al. ${ }^{10}$ recently showed the theoretical relationship between the DMI and Hund's rule in $\mathrm{Co} / \mathrm{X}$ and other $3 \mathrm{~d} / 5 \mathrm{~d}$ bilayer structures. Torrejon et al. ${ }^{11}$ reported the dependence of the DMI on materials in NM/ $\mathrm{CoFeB} / \mathrm{MgO}(\mathrm{NM}=\mathrm{Hf}, \mathrm{Ta}$, and $\mathrm{W})$ with the nitrification of the Ta layer. Yu et al. ${ }^{12}$ provided a way to resolve the contributions on the DMI at each $\mathrm{Pt} / \mathrm{Co}$ and $\mathrm{Co} / \mathrm{X}$ interface in a $\mathrm{Pt} / \mathrm{Co} /[\mathrm{Ni} / \mathrm{Co}]_{4} / \mathrm{X}$ multilayer structure. Kim et al. ${ }^{13}$ revealed the DMI-induced asymmetry of the DW speed in a $\mathrm{Pt} / \mathrm{Co} / \mathrm{X}$ trilayer system. Since the physical origin of the DMI at a metallic interface still remains elusive, an experimental study of the key intrinsic parameters of the DMI will be of great help and can provide an empirical guideline in terms of both experiment and sample structure design for application and to help understand the DMI.

Therefore, we are interested in the DMI at interfaces between ferromagnetic metal and nonmagnetic metals in a bilayer system. The interfacial DMI in such metallic

\section{(c) The Author(s) 2018}

(c) (i) Open Access This article is licensed under a Creative Commons Attribution 4.0 International License, which permits use, sharing, adaptation, distribution and reproduction cc) in any medium or format, as long as you give appropriate credit to the original author(s) and the source, provide a link to the Creative Commons license, and indicate if changes were made. The images or other third party material in this article are included in the article's Creative Commons license, unless indicated otherwise in a credit line to the material. If material is not included in the article's Creative Commons license and your intended use is not permitted by statutory regulation or exceeds the permitted use, you will need to obtain permission directly from the copyright holder. To view a copy of this license, visit http://creativecommons.org/licenses/by/4.0/. 
bilayers arises from a three-site indirect exchange mechanism among two atomic spins and a neighboring atom with a large spin-orbit coupling (SOC) ${ }^{2,4,14}$ In antiferromagnetic crystals, the DMI is known to originate from an anisotropic superexchange interaction that linearly depends on the strength of the SOC. ${ }^{4}$ In this case, the DMI magnitude is proportional to $(\Delta g / g) J_{\mathrm{ex}}$, where $g$ is the gyromagnetic ratio, $\Delta g$ is its deviation from the value of a free electron, and $J_{\mathrm{ex}}$ is related to the magnitude of the exchange interaction. ${ }^{4}$

Whether associating this elegant picture to polycrystalline metallic systems is possible is an interesting question. Spin-glass alloy systems shed light on the origin of the DMI in metallic systems in which the DMI arises from the spin-orbit scattering of conducting electrons by nonmagnetic transition-metal impurities. ${ }^{14}$ This condition inspired us to investigate the material parameters that may be correlated with the scattering potential at the metal-metal interface. If we can find a correlation between the DMI strength and other material parameters, such as the work function, electronegativity, and SOC constant, this would provide a productive guideline for engineering the DMI strength. In this paper, we report an experimental observation of the correlation between the DMI strength and the work function difference at the metal-metal interface.

\section{Materias and methods}

\section{Sample fabrication and detail structure}

The detailed film structure is $5 \mathrm{~nm} \mathrm{Ta} / 2.5 \mathrm{~nm} \mathrm{Pt} / 0.9 \mathrm{~nm}$ $\mathrm{Co} / 2.5 \mathrm{~nm} \mathrm{X} / 1.5 \mathrm{~nm} \mathrm{Pt}$, where $\mathrm{X}$ was selected as $\mathrm{Ti}, \mathrm{Cu}$, $\mathrm{W}, \mathrm{Ta}, \mathrm{Al}, \mathrm{Ru}, \mathrm{Pd}, \mathrm{Au}$, or Pt, which was deposited by dcmagnetron sputtering on $\mathrm{Si}$ wafers with a $300 \mathrm{~nm} \mathrm{SiO}_{2}$ layer. This structure was chosen to observe the relative DMI tendency of bilayer systems while keeping the bottom Pt layer and Co thickness the same. The lowermost
Ta layer is a seed layer used to enhance the crystallinity of the films, and the uppermost Pt layer is a protective layer used to prevent oxidation. To keep the quality of the Pt/ $\mathrm{Co} / \mathrm{X}$ films as similar as possible and to exclude other unknown factors that affect the depinning field measurement, the sputtering conditions (Ar working pressure $\sim 2 \mathrm{mTorr}$, sputtering power $\sim 10 \mathrm{~W}$ ) were carefully kept the same for all $\mathrm{Pt} / \mathrm{Co} / \mathrm{X}$ films. High-resolution transmission electron microscopy ${ }^{15}$ revealed that the Pt layer has an fcc (111) crystalline structure along the growth direction, ${ }^{16}$ which is known to exhibit a strong perpendicular magnetic anisotropy (PMA). ${ }^{17-19}$ The overall film roughness, $R$, was measured via atomic force microscopy as listed in Table 1. No clear grain structure was observed, possibly due to the thin thickness of our films. ${ }^{16}$ The depinning fields, $H_{\mathrm{P}}$, are also listed in Table 1 . These measurements confirm that there is no significant variation in both $R$ and $H_{\mathrm{P}}$ among all the samples.

\section{Measurement of the SOT efficiency to quantify the DMI of the trilayer-structured samples}

To measure the SOT efficiency, $\varepsilon$, and the $H_{\mathrm{DMI}}$, which is the DMI-induced effective magnetic field, the samples were patterned to $20 \mu \mathrm{m}$-wide and $350 \mu \mathrm{m}$-long microwires by photolithography and an ion-milling process, as shown in Fig. 1a. The $\varepsilon$ and $H_{\mathrm{DMI}}$ were measured from the depinning field of the DWs with respect to the in-plane magnetic field, $H_{x}{ }^{15,20,21}$ The measurement procedure is described as follows. First, a large perpendicular external magnetic field was applied to saturate the magnetization of the sample either up or down. Next, a DW was created at the position inside the microwire and adjacent to the DW writing electrode, as shown by the white vertical line in Fig. 1a. Then, under application of a fixed current bias, an out-of-plane magnetic field was swept until the DW moved from the initial position. By repeating this

Table $1 H_{\mathrm{DMl}}, H_{\mathrm{K}}, K_{\mathrm{eff}}, D, W_{\text {mea }} R$, and $H_{\mathrm{P}}$ for the $\mathrm{Pt} / \mathrm{Co} / \mathrm{X}$ samples with material $\mathrm{X}$

\begin{tabular}{llllllll}
\hline $\mathbf{X}$ & $\boldsymbol{H}_{\mathrm{DMI}}[\mathrm{mT}]$ & $\boldsymbol{H}_{\mathbf{K}}[\mathrm{T}]$ & $\boldsymbol{K}_{\text {eff }}\left[10^{5} \mathrm{~J} / \mathbf{m}^{3}\right]$ & $\boldsymbol{D}\left[\mathrm{mJ} / \mathbf{m}^{2}\right]$ & $\boldsymbol{W}_{\text {mea }}[\mathrm{eV}]$ & $R[\mathrm{~nm}]$ & $\boldsymbol{H}_{\mathbf{P}}[\mathrm{mT}]$ \\
\hline $\mathrm{Ti}$ & $-197 \pm 25$ & $1.13 \pm 0.013$ & $7.94 \pm 0.09$ & $-1.45 \pm 0.18$ & $3.66 \pm 0.052$ & 0.80 & $13.0 \pm 0.2$ \\
$\mathrm{Cu}$ & $-190 \pm 25$ & $0.90 \pm 0.021$ & $6.28 \pm 0.15$ & $-1.58 \pm 0.19$ & $4.37 \pm 0.007$ & 0.47 & $17.5 \pm 0.3$ \\
$\mathrm{~W}$ & $-183 \pm 5$ & $0.95 \pm 0.012$ & $6.62 \pm 0.08$ & $-1.48 \pm 0.03$ & $4.77 \pm 0.002$ & 0.59 & $11.1 \pm 0.2$ \\
$\mathrm{Ta}$ & $-160 \pm 10$ & $1.20 \pm 0.018$ & $8.37 \pm 0.13$ & $-1.15 \pm 0.06$ & $4.01 \pm 0.073$ & 0.67 & $13.6 \pm 0.2$ \\
$\mathrm{Al}$ & $-109 \pm 5$ & $0.94 \pm 0.049$ & $6.61 \pm 0.35$ & $-0.88 \pm 0.02$ & $3.72 \pm 0.034$ & 0.54 & $16.0 \pm 0.7$ \\
$\mathrm{Ru}$ & $-51 \pm 4$ & $0.65 \pm 0.006$ & $4.54 \pm 0.04$ & $-0.49 \pm 0.04$ & $4.67 \pm 0.098$ & 0.68 & $10.8 \pm 0.3$ \\
$\mathrm{Pd}$ & $-43 \pm 4$ & $0.46 \pm 0.006$ & $3.24 \pm 0.04$ & $-0.49 \pm 0.04$ & $5.23 \pm 0.034$ & 0.58 & $16.8 \pm 0.4$ \\
$\mathrm{Au}$ & $-32 \pm 5$ & $0.75 \pm 0.006$ & $5.22 \pm 0.04$ & $-0.29 \pm 0.04$ & $5.30 \pm 0.061$ & 0.75 & $13.8 \pm 0.2$ \\
$\mathrm{Pt}$ & $0 \pm 10$ & $0.79 \pm 0.010$ & $5.51 \pm 0.07$ & $0.00 \pm 0.09$ & $5.30 \pm 0.098$ & 0.93 & $13.7 \pm 0.2$ \\
\hline
\end{tabular}

Here, the film roughness, $R$, was obtained via the standard deviation of the surface height, and $H_{\mathrm{p}}$ was measured when $J$ and $H_{\mathrm{x}}$ were zero. The $W_{\text {mea }}$ of $C_{0}$ is $4.50 \pm$ $0.127 \mathrm{eV}$. 


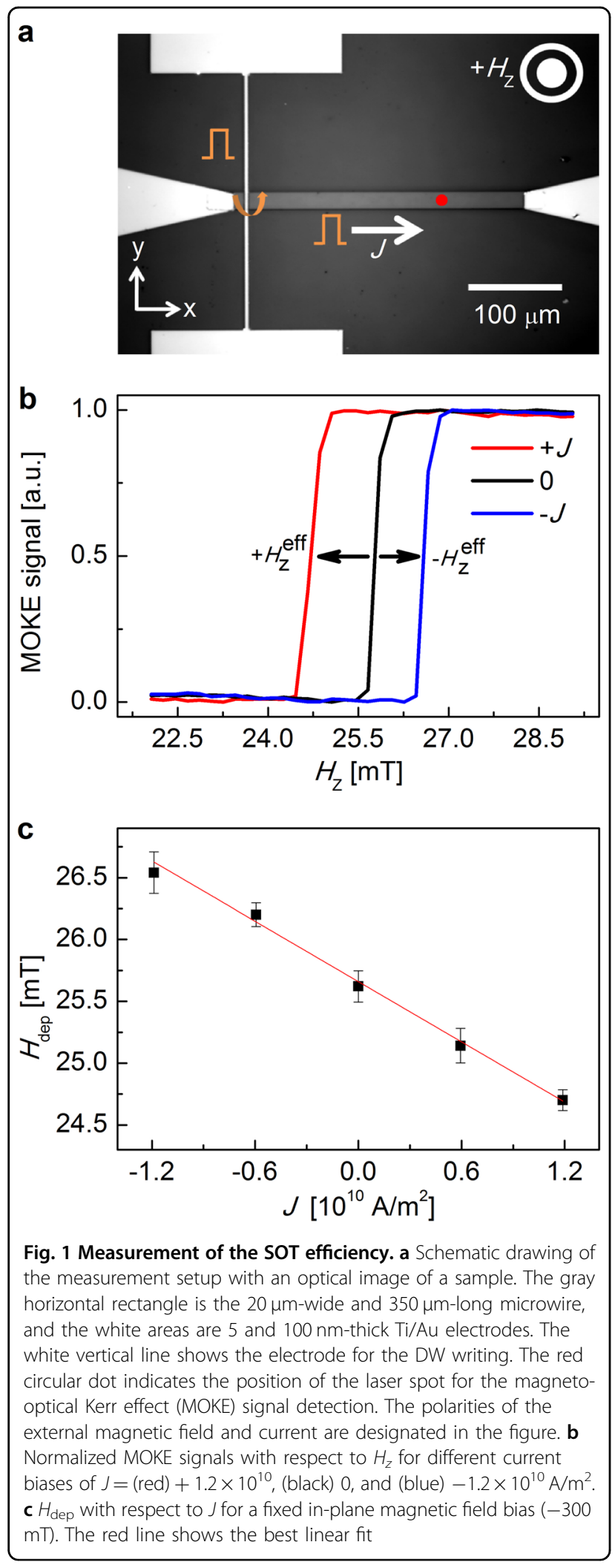

procedure using different magnitudes of the current bias as shown in Fig. 1b, the depinning field, $H_{\text {dep }}$, was measured as a function of the total current density, J. From the linear dependence of $H_{\text {dep }}$ on $J$, as shown in Fig. 1c, we quantified $\varepsilon$ using the relation $\varepsilon=-\partial H_{\mathrm{dep}} / \partial J^{15,20,21}$ The measurement was repeated for different $H_{x}$ values.

Based on the SOT theory, it is known that the dampinglike torque has the direction parallel to $\hat{m} \times(\hat{m} \times \hat{y}),{ }^{5}$ which corresponds to a situation in which there exists an effective magnetic field parallel to the $\hat{m} \times \hat{y}$ term. Here, $\hat{m}$ is the magnetization vector inside a DW. The microwire lies on the $x-y$ plane, and the current flows along the $x$ axis. Therefore, the finite $x$-component of $\hat{m}$ generates the $z$-component of $\hat{m} \times \hat{y}$, i.e., the perpendicular component of the SOT-induced effective magnetic field. This perpendicular component of the SOT-induced effective magnetic field assists the DW depinning process. For the case of a Bloch-type DW, due to the zero $x$-component of $\hat{m}$, there is no $z$-component for the effective magnetic field, and thus $\varepsilon=0$. Hence, the intercept to the $x$-axis in Fig. 2 indicates that the DW stays in the Bloch-type DW configuration.

Figure 2 shows the plot of $\varepsilon$ with respect to $H_{x}$ for the samples with different $X$ values, as denoted in each panel. All the plots of $\varepsilon$ exhibit three distinct regimes that are frequently observed in SOT-induced DW motions: two saturation regimes of the Néel-type DW configurations and a transition regime in between. ${ }^{22}$ In the transition regime, a Bloch-type DW configuration appeared at the intercept to the abscissa (as shown by the red vertical lines) in which magnetic field $H_{x}^{0}$ exactly compensated for $H_{\text {DMI }}$ (i.e., $H_{x}^{0}+H_{D M I}=0$ ). We could, therefore, quantify $H_{\mathrm{DMI}}$ from these measurements. All the samples with broken inversion symmetry exhibited nonzero $H_{\mathrm{DMI}}$, except the sample with $\mathrm{X}=\mathrm{Pt}$, which had an almost zero $H_{\mathrm{DMI}}$ because of its symmetrically layered structure.

The $\varepsilon$ corresponds to the overall SOT efficiency with respect to $J$, where $J$ is defined as the total current divided by the section of the wire. Since $\varepsilon$ is composed of the contributions from the upper $\mathrm{X}$ and lower Pt layers, of which the local current densities are different to each other due to their different conductivities, there may be several other ways to define the current density. ${ }^{23}$ However, a different definition of $J$ changes only the scale of the ordinate in Fig. 2, whereas the intercept to the abscissa (i.e., $H_{x}^{0}$ ) can be uniquely determined. Of note, the DMI can be determined without current injection (i.e., no SOT phenomena) by purely field-induced DW motion or spin wave propagation. $^{24,25}$ 


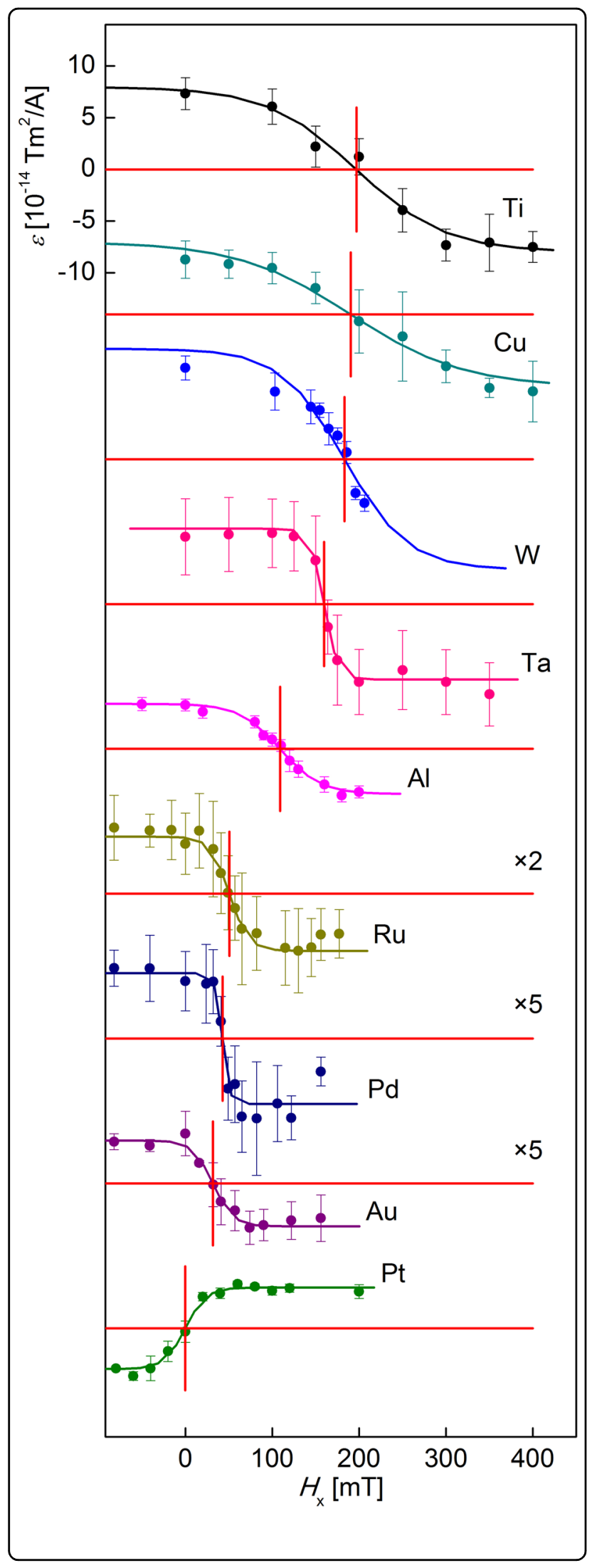

Fig. 2 SOT efficiency of $\mathrm{Pt} / \mathrm{Co} / \mathbf{X}$. Plots of $\varepsilon$ with respect to $H_{x}$ for different $X$ values as denoted in the figures. The curved solid lines are best fits to guide the eye. The red horizontal lines show the axis of $\varepsilon=$ 0 for each measurement. The red vertical lines indicate the positions of $H_{x}^{0}$ for $\varepsilon=0$. The values of $\varepsilon$ for $\mathrm{X}=\mathrm{Ru}, \mathrm{Pd}$, and $\mathrm{Au}$ are magnified for better resolution of the data with amplification factors denoted in the figure

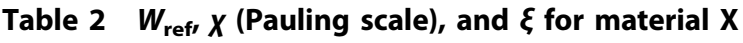

\begin{tabular}{lllll}
\hline $\mathbf{X}$ & $\boldsymbol{W}_{\text {ref }}[\mathrm{eV}]$ & $\boldsymbol{X}^{\mathrm{a}}$ [Pauling] & $\begin{array}{l}\boldsymbol{\xi}^{\mathbf{b}}\left[\mathbf{1 \mathbf { 0 } ^ { \mathbf { 4 } }}\right. \\
\left.\mathbf{m}^{-\mathbf{1}}\right]\end{array}$ & References for $\boldsymbol{W}_{\text {ref }}$ \\
\hline $\mathrm{Ti}$ & $3.67 \pm 0.67$ & 1.5 & 1.2 & $36,42,55$ \\
$\mathrm{Cu}$ & $4.65 \pm 0.20$ & 1.9 & 8.6 & $36,42,43,45,51,53$ \\
$\mathrm{~W}$ & $4.8 \pm 0.45$ & 2.4 & 24.3 & $36,39-42,45,50$ \\
$\mathrm{Ta}$ & $4.37 \pm 0.31$ & 1.5 & 19.7 & $36-39,42,45,46$ \\
$\mathrm{Al}$ & $4.23 \pm 0.16$ & 1.6 & 0.6 & $36,39,42,43,45,47,49,56$ \\
$\mathrm{Ru}$ & $4.85 \pm 0.27$ & 2.2 & 10.4 & $36,42,45$ \\
$\mathrm{Pd}$ & $5.35 \pm 0.29$ & 2.2 & 15.0 & $36,42,44,45,48$ \\
$\mathrm{Au}$ & $5.17 \pm 0.30$ & 2.5 & 51.0 & $36,39,42,45,49,52,55$ \\
$\mathrm{Pt}$ & $5.68 \pm 0.24$ & 2.5 & 44.8 & $36,42,45$ \\
\hline
\end{tabular}

The values and the error bars of $W_{\text {ref }}$ were calculated as the means and standard deviations of values from refs. ${ }^{33-56}$. The $W_{\text {ref }}$ of $C_{0}$ is $4.69 \pm 0.27 \mathrm{eV}^{33-36,42}$ Superscripts ${ }^{\mathrm{a}}$ and ${ }^{\mathrm{b}}$ indicate relevant refs. ${ }^{57,58}$, respectively.

\section{Results and discussion}

Correlations between the material parameters and the DMI

Tables 1 and 2 list the measured values of $H_{\mathrm{DMI}}$, anisotropy field, $H_{\mathrm{K}}$, effective uniaxial magnetic anisotropy, $K_{\text {eff }}$, DMI strength, $D$, work function, $W_{\text {mea }}$ measured by ultraviolet photoelectron spectroscopy (UPS), overall sample roughness, $R$, depinning field, $H_{\mathrm{p}}$, and the literature values of the work function, $W_{\text {ref }}$, electronegativity, $\chi$, and SOC constant, $\xi$. These material parameters were chosen because of their potential relationship with the electrostatic potential barrier at the interface between the $\mathrm{Co}$ and $\mathrm{X}$ layers. In addition, the correlation between these easily accessible bulk values may be better as a practical guideline for the selection of the top and bottom non-magnetic layers from the literature. From the tables, we see that $H_{\mathrm{DMI}}$ has a better correlation with $W$ than the other parameters. The correlations between $W, \chi$, and $\xi$ are plotted and shown in Fig. 3, where the $W_{\text {mea }}$ values are denoted by red symbols and the $W_{\text {ref }}$ values are black symbols, which confirm that a better correlation exists in Fig. 3a than in the other figures.

More specific and precise measurements would provide a more accurate relationship between the DMI and work function. Since the present $W_{\text {mea }}$ was measured after 


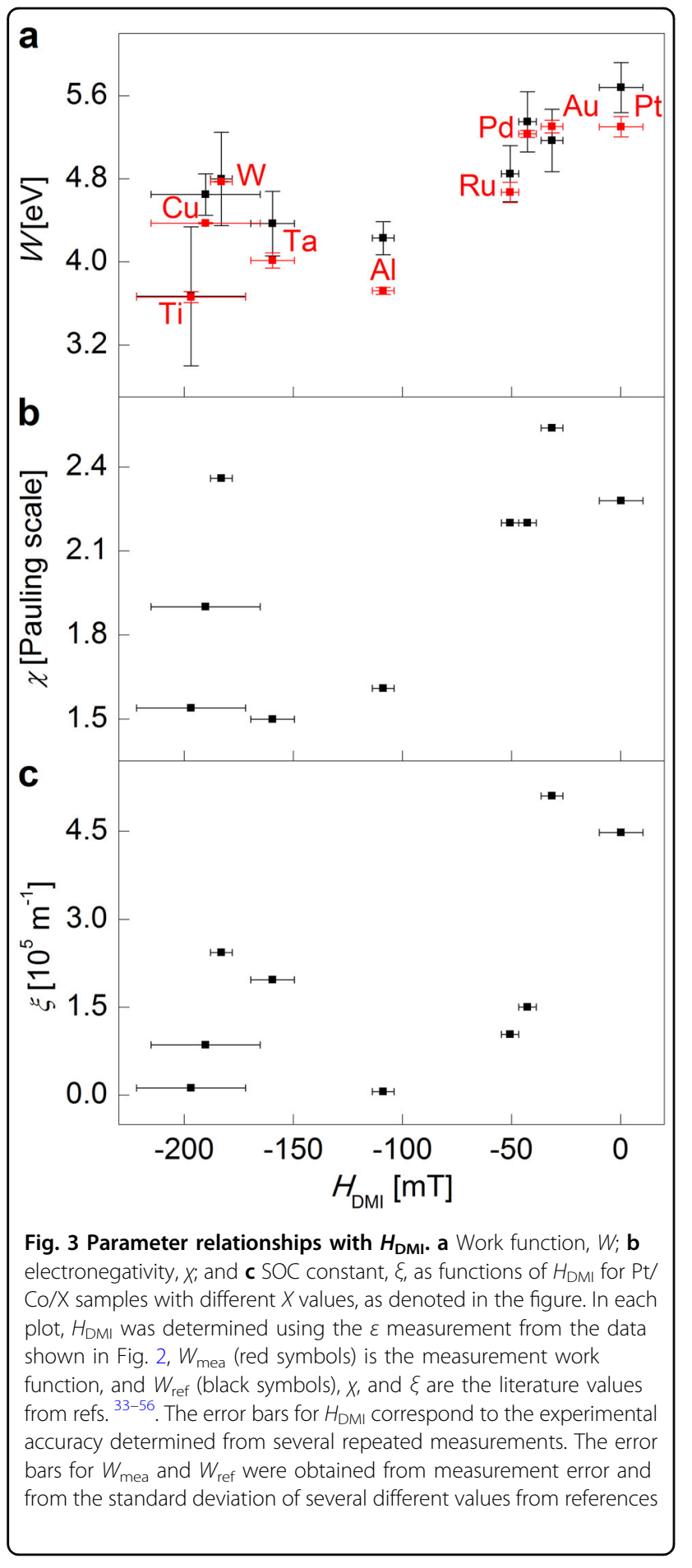

in situ surface cleaning on relatively thick X layers, there may be artefacts, such as crystal deformation, induced strain, and atomic mixing, which formed during the surface cleaning process. Additionally, since the $\mathrm{X}$ layers must be thicker than the penetration depth of the UPS measurement, accurate information at the vicinity of the interface might not have been precisely collected. Despite these experimental limits and possible artefacts, our observation suggests the possibility that the work function may play a more significant role in the generation of $H_{\text {DMI }}$.

Based on the concept of the potential gradient at the interface, the electronegativity may also have a relationship with the DMI, as shown in Fig. $3 \mathrm{~b}$, with a rough correlation between $X$ and $H_{\mathrm{DMI}}$. Although the electronegativity difference between $\mathrm{Co}$ and $\mathrm{X}$ also implies a potential gradient at the interface, it is relevant to atomic and/or molecular systems, but less relevant to metallic bilayer system because it is associated with the chemical energy of the valence bond. In addition, because the spin-orbit scattering-mediated spin-chiral effect may play a leading role in our trilayer system, our $\mathrm{Pt} / \mathrm{Co} / \mathrm{X}$ metallic system shows a better relationship between the DMI and work function compared with the DMI and electronegativity.

Another important factor in determining the strength of the DMI is the SOC. The strength, $D$, of the DMI could be linearly proportional to the strength of the SOC, which is similar to that in antiferromagnetic crystals. ${ }^{4}$ However, the experimental correlation between $H_{\mathrm{DMI}}$ and $\xi$ was found to be pretty scattered, as shown in Fig. 3c. Because our values of $\xi$ correspond to the atomic SOCs from the literature, if more specific and precise SOC values relevant to the metallic bilayer systems were available, we may possibly see a more accurate relationship between the DMI and SOC.

The magnitude of $D$ was estimated from the relation of $D=\mu_{0} H_{\mathrm{DMI}} M_{\mathrm{S}} \lambda$ with a Bloch-type DW width of $\lambda\left(=\sqrt{A / K_{\text {eff }}}\right), 5,7,26$ where $A$ is the exchange stiffness. The Co value of $A\left(2.2 \times 10^{-11} \mathrm{~J} / \mathrm{m}\right)$ and $M_{\mathrm{S}}$ of $\mathrm{Co}(1.4 \times$ $10^{6} \mathrm{~A} / \mathrm{m}$ ) were used in the estimation for a qualitative comparison. ${ }^{26,27}$ The effective anisotropy was quantified from the relation $K_{\text {eff }}=M_{\mathrm{S}} H_{\mathrm{K}} / 2$. The $D$ was defined as the total effective DMI, which includes contributions from the upper $\mathrm{Pt} / \mathrm{Co}$ and lower $\mathrm{Co} / \mathrm{X}$ interfaces. The scattering potential barrier was presumed to be associated with the work function difference, $\Delta W$, at the $\mathrm{Co} / \mathrm{X}$ interface. Figure 4 shows a summary of the $D$ values as a function of $\Delta W_{\text {mea }}\left(\equiv W_{\text {mea, } \mathrm{X}}-W_{\text {mea,Co }}\right)$ and $\Delta W_{\text {ref }}\left(\equiv W_{\text {ref, } \mathrm{X}}-W_{\text {ref, }}\right.$ Co), where $\Delta W_{\text {mea }}$ values are denoted by red symbols measured via UPS and $\Delta W_{\text {ref }}$ values are shown as black symbols, which were obtained from the literature. The correlation between $D$ and $\Delta W$ is better than between $H_{\text {DMI }}$ and $W$.

Reference $^{14}$ described how to calculate the magnitude of the DMI for nonmagnetic transition-metal impurities ( $\mathrm{Ti}, \mathrm{Ni}, \mathrm{Pd}, \mathrm{Fe}, \mathrm{Co}$, and $\mathrm{Pt}$ ) in CuMn spin-glass alloys. The DMI is associated with "the shift in the ground state energy of gas of conduction electrons interacting with two

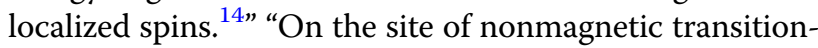
metal impurities, the spin-orbit coupling of a conduction 


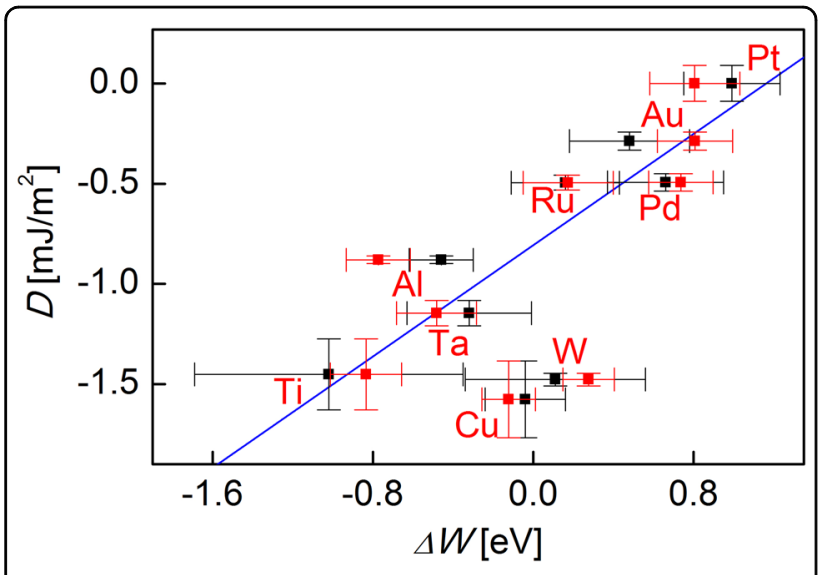

Fig. 4 Plot of $D$ as a function of $\Delta W$ for the $\mathrm{Pt} / \mathrm{Co} / \mathrm{X}$ samples with different $X$ values, as denoted near the data symbols. The $\Delta W_{\text {mea }}$ values are denoted by red symbols, and $\Delta W_{\text {ref }}$ values are shown by black symbols. The blue line shows the best linear fit. The error bars for $D$ were obtained from the statistical summation of the experimental accuracy of $H_{D M I}$ and $H_{K}$ determination. The error bars for $W_{\text {mea }}$ are from measurement errors, and those for $W_{\text {ref }}$ were obtained from the standard deviation of several different values from references

electron is considerably enhanced because the admixture of the impurity $d$ states into the conduction band allows the conduction electrons to experience the strong spin-orbit forces of the $d$ states. ${ }^{14}$ " They showed that the DMI is due to spin-orbit scattering of conduction electrons by nonmagnetic impurities, and the strength of the DMI is proportional to the magnitude of the scattering potential. ${ }^{14}$ In the FM/HM bilayer systems, such as those in our experiments, the work function difference between the two metals provides such a scattering potential. When two metals with different work functions are brought into contact, the Fermi energy levels of two metals are aligned to build a very narrow potential barrier within the Thomas-Fermi length. The height of this potential barrier is proportional to the work function difference of the two metals. Consequently, the strength of the DMI may be related to the work function difference between two metals.

The signs of the DMI are all negative for the samples of the $\mathrm{Pt} / \mathrm{Co} / \mathrm{X}$ trilayer structure, except for $\mathrm{X}=\mathrm{Pt}$. The negative DMI generates left-handed chiral DWs. ${ }^{20}$ For the case of $\mathrm{X}=\mathrm{Pt}$, a negligibly small DMI was expected due to its symmetric structure; however, several recent studies have reported a small but positive DMI, which is possibly due to the different interfaces that form between $\mathrm{Pt} / \mathrm{Co}$ and $\mathrm{Co} / \mathrm{Pt}^{24,28}$ Our observation of negative DMIs in the other samples is in agreement with other studies for the $\mathrm{Pt} / \mathrm{Co} / \mathrm{Pd}{ }^{29} \mathrm{Pt} / \mathrm{Co} / \mathrm{Al},{ }^{30} \mathrm{Pt} / \mathrm{Co} / \mathrm{Cu},{ }^{30} \mathrm{Pt} / \mathrm{Co} / \mathrm{Ta},{ }^{31} \mathrm{Pt} / \mathrm{Co} /$ $[\mathrm{Ni} / \mathrm{Co}]_{4} / \mathrm{Cu},{ }^{12}$ and $\mathrm{Pt} / \mathrm{Co} /[\mathrm{Ni} / \mathrm{Co}]_{4} / \mathrm{Ta}^{12}$ films after consideration of sign conventions. It is known that a strong $\mathrm{SOC}$ exists at the Pt/Co interface, and consequently, the
DMI strength is very large at the $\mathrm{Pt} / \mathrm{Co}$ bilayer interface. ${ }^{10,32}$ From these results, we infer that the sign of the DMI may be determined in our trilayer samples by the underlayer Pt. Because our measurement is the sum of the effects on the DMI of the two interfaces, the effect of each interface cannot be independently observed. Even if the underlayer Pt is dominant in the total DMI, directly knowing the sign and magnitude of the DMI at the $\mathrm{Co} / \mathrm{X}$ bilayer interface is difficult. However, the relative strength of the $\mathrm{DMI}$ between the $\mathrm{Pt} / \mathrm{Co} / \mathrm{X}$ trilayers remains significant.

In summary, we presented experimental observations regarding the correlation between the DMI strength and the work function. This correlation may be related to the spin-orbit scattering in the electric potential barrier due to the work function difference at the interfaces. This correlation suggests that the DMI strength can be engineered via material selection following guidelines related to the intrinsic material parameters.

\section{Acknowledgments}

This work was supported by grants from the National Research Foundations of Korea (NRF) funded by the Ministry of Science, ICT and Future Planning of Korea (MSIP) (2015R1A2A1A05001698 and 2015M3D1A1070465). Y.-K.P. and B.-

C.M. were supported by the National Research Council of Science and Technology (NST) (Grant no. CAP-16-01-KIST) by the Korean government (MSIP). We thank Seong-Hyub Lee for sample preparation for the UPS measurement.

\section{Publisher's note}

Springer Nature remains neutral with regard to jurisdictional claims in published maps and institutional affiliations.

Received: 11 October 2017 Revised: 2 July 2018 Accepted: 21 August 2018. Published online: 12 October 2018

\section{References}

1. Parkin, S. S. P. \& Yang, S.-H. Memory on the racetrack. Nat. Nanotechnol. 10, 195-198 (2015).

2. Fert, A., Cros, V. \& Sampaio, J. Skyrmions on the track. Nat. Nanotechnol. 8, 152-156 (2013).

3. Dzyaloshinsky, I. A thermodynamics theory of weak ferromagnetism of antiferromagnetics. J. Phys. Chem. Solid 4, 241-255 (1958).

4. Moriya, T. Anisotropic superexchange interaction and weak ferromagnetism. Phys. Rev. 120, 91-98 (1960).

5. Emori, S., Bauer, U., Ahn, S.-M., Martinez, E. \& Beach, G. S. D. Current-driven dynamics of chiral ferromagnetic domain walls. Nat. Mater. 12, 611-616 (2013).

6. Ryu, K-S., Thomas, L., Yang, S.-H. \& Parkin, S. S. P. Chiral spin torque at magnetic domain walls. Nat. Nanotechnol. 8, 527-533 (2013).

7. Thiaville, A.r Rohart, S., Jué, É., Cros, V. \& Fert, A. Dynamics of Dzyaloshinskii domain walls in ultrathin magnetic films. Europhys. Lett. 100, 57002 (2012).

8. Sampaio, J., Cros, V., Rohart, S., Thiaville, A. \& Fert, A. Nucleation, stability and current-induced motion of isolated magnetic skyrmions in nanostructures. Nat. Nanotechnol. 8, 839-844 (2013).

9. Yoo, J.-W., Lee, S.-J., Moon, J.-H. \& Lee, K.J. Phase diagram of a single skyrmion on magnetic nanowires. IEEE Trans. Magn. 50, 1500504 (2014).

10. Belabbes, A., Bihlmayer, G., Bechstedt, F., Blügel, S. \& Manchon, A. Hund's ruledriven Dzyaloshinskii-Moriya interaction ad 3d-5d interfaces. Phys. Rev. Lett. 117, 247202 (2016)

11. Torrejon, J. et al. Interface control of the magnetic chirality in CoFeB/MgO heterostructures with heavy-metal underlayers. Nat. Communun. 5, 4655 (2014). 
12. $Y u$, J. et al. Spin-orbit torques and Dzyaloshinskii-Moriya interaction in dualinterfaced Co-Ni multilayers. Sci. Rep. 6, 32629 (2016).

13. Kim, D.-Y. et al. Chirality-induced antisymmetry in magnetic domain-wall speed. NPG Asia Mater. 10, e464 (2018).

14. Fert, A. \& Levy, P. M. Role of anisotropic exchange interactions in determining the properties of spin-glasses. Phys. Rev. Lett. 44, 1538-1541 (1980).

15. Je, S.-G. et al. Emergence of huge negative spin-transfer torque in atomically thin Co layers. Phys. Rev. Lett. 118, 167205 (2017).

16. Wellhőfer, M., Weißenborn, M., Anton, R., Pütter, S. \& Oepen, H. P. Morphology and magnetic properties of ECR ion beam sputtered Co/Pt films. J. Magn. Magn. Mater. 292, 345-358 (2005).

17. Carcia, P. F., Li, Z. G. \& Zeper, W. B. Effect of sputter-deposition processes on the microstructure and magnetic properties of Pt/Co multilayers. J. Magn. Magn. Mater. 121, 452-460 (1993).

18. Carcia, P. F., Reilly, M. \& Li, Z. G. Ar-sputtered Pt/Co multilayers with large anisotropy energy and coercivity. IEEE Trans. Magn. 30, 4395-4397 (1994).

19. Canedy, C. L., Li, X. W. \& Xiao, G. Extraordinary Hall effect in (111) and (100)orientated Co/Pt superlattices. J. Appl. Phys. 81, 5367 (1997).

20. Emori, S. et al. Spin Hall torque magnetometry of Dzyaloshinskii domain walls. Phys. Rev. B 90, 184427 (2014).

21. Franken, J. H., Herps, M., Swagten, H. J. M. \& Koopmans, B. Tunable chiral spin texture in magnetic domain walls. Sci. Rep. 4, 5248 (2014).

22. Haazen, P. P. J. et al. Domain-wall depinning governed by the spin Hall effect. Nat. Mater. 12, 299-303 (2013)

23. Yu, J., Qiu, X., Legrand, W. \& Yang, H. Large spin-orbit torques in Pt/Co-Ni/W heterostructures. Appl. Phys. Lett. 109, 042403 (2016).

24. Je, S.-G. et al. Asymmetric magnetic domain-wall motion by the Dzyalishinskii-Moriya interaction. Phys. Rev. B 88, 214401 (2013).

25. Cho, J. et al. Thickness dependence of the interfacial Dzyaloshinskii-Moriya interaction in inversion symmetry broken systems. Nat. Commun. 6, 7635 (2015).

26. Metaxas, P. J. et al. Creep and flow regimes of magnetic domain-wall motion in ultrathin Pt/Co/Pt films with perpendicular anisotropy. Phys. Rev. Lett. 99, 217208 (2007)

27. Bandiera, S., Sousa, R. C., Rodmacq, B. \& Dieny, B. Asymmetric interfacial perpendicular magnetic anisotropy in Pt/Co/Pt trilayers. IEEE Magn. Lett. 2, 3000504 (2011)

28. Lavrijsen, R. et al. Asymmetric magnetic bubble expansion under in-plane field in Pt/Co/Pt: effect of interface engineering. Phys. Rev. B 91, 104414 (2015).

29. Guan, Y., Koyama, T. \& Chiba, D. Current-induced magnetic domain-wall motion in $\mathrm{Pt} / \mathrm{Co} / \mathrm{Pd}$ and $\mathrm{Pd} / \mathrm{Co} / \mathrm{Pt}$ structures with opposite sign of Dzyaloshinskii-Moriya interaction. AIP Adv. 7, 085123 (2017).

30. Ajejas, F. et al. Tuning domain-wall velocity with Dzyaloshinskii-Moriya interaction. Appl. Phys. Lett. 111, 202402 (2017).

31. Cho, J. et al. The sign of the interfacial Dzyaloshinskii-Moriya interaction in ultrathin amorphous and polycrystalline magnetic films. J. Phys. D 50, 425004 (2017).

32. Yang, H., Thiaville, A., Rohart, S., Fert, A. \& Chshiev, M. Anatomy of Dzyalishinskii-Moriya interaction at Co/Pt interfaces. Phys. Rev. Lett. 115 267210 (2015)

33. Hall, G. K. \& Mee, C. H. B. The surface potential of oxygen on iron, cobalt and manganese. Surf. Sci. 28, 598-606 (1971).
34. Wahlin, H. B. Thermionic properties of the iron group. Phys. Rev. 61, 509-512 (1942).

35. Yu, A. Y.-C. \& Spicer, W. E. Photoemission studies of the electronic structure of cobalt. Phys. Rev. 167, 674-167 (1968).

36. Michaelson, H. B. The work function of the elements and its periodicity. J. Appl. Phys. 48, 4729-4733 (1977).

37. Cardwell, A. B. The thermionic properties of tantalum. Phys. Rev. 47, 628-630 (1935).

38. Kiejna, A. Surface atomic structure and energetics of tantalum. Surf. Sci. 598, 276-284 (2005).

39. Hopkins, B. J. \& Rivière, J. C. Work function values from contact potentia difference measurements. Br. J. Appl. Phys. 15, 941-946 (1964).

40. Davisson, C. \& Germer, L. H. The thermionic work function of tungsten. Phys. Rev. 20, 300-330 (1922)

41. Todd, C. J. \& Rhodin, T. N. Work function in field emission - the (110) plane of tungsten. Surf. Sci. 36, 353-369 (1973).

42. Lide, D. R. CRC Handbook of Chemistry and Physics. 75th edn, (CRC Press, Boca Raton, FL, USA, 1994).

43. Mitchell, E. W. J. \& Mitchell, J. W. The work functions of copper, silver and aluminium. Proc. R. Soc. A210, 70-84 (1951).

44. Smith, G. C., Norris, C., Binns, C. \& Padmore, H. A. A photoemission study of ultrathin palladium overlayers on low-index faces of silver. J. Phys. C15 6481-6496 (1982)

45. Kawano, H. Effective work functions for ionic and electronic emissions from mono- and polycrystalline surfaces. Prog. Surf. Sci. 83, 1-165 (2008).

46. Shelton, $\mathrm{H}$. Thermionic emission from a planar tantalum crystal. Phys. Rev. 107 1553-1557 (1957)

47. Grepstad, J. K., Gartland, P. O. \& Slagsvold, B. J. Anisotropic work function of clean and smooth low-index faces of aluminium. Surf. Sci. 57, 348-362 (1976).

48. Dubridge, L. A. \& Roehr, W. W. Photoelectric and thermionic properties of palladium. Phys. Rev. 39, 99-107 (1932).

49. Uda, M., Nakamura, A., Yamamoto, T. \& Fujimoto, Y. Work function of polycrystalline Ag, Au, and Al. J. Electron Spectrosc. Relat. Phenom. 88, 643-648 (1998).

50. Camp, M. \& Lecchini, S. M. A. The work function of polycrystalline tungsten foil. Proc. Phys. Soc. 85, 815-817 (1965)

51. Anderson, P. A. The work function of copper. Phys. Rev. 76, 388-390 (1949).

52. Anderson, P. A. Work function of gold. Phys. Rev. 115, 553-554 (1959).

53. Gartland, P. O., Berge, S. \& Slagsvold, B. J. Photoelectric work function of a copper single crystal for the (100), (110), (111), and (112) faces. Phys. Rev. Lett. 28, 738-739 (1972).

54. Bouwman, R. \& Sachtler, W. M. H. Photoelectric determination of the work function of gold-platinum alloys. J. Catal. 19, 127-140 (1970).

55. D'Arcy, R. J. \& Surplice, N. A. Work function of titanium films. Surf. Sci. 36 783-788 (1973).

56. Eastment, R. M. \& Mee, C. H. B. Work function measurements on (100), (110) and (111) surfaces of aluminium. J. Phys. F3, 1738-1745 (1973).

57. Huheey, J. E., Keiter, E. A. \& Keiter, R. L. Inorganic Chemistry. 4th edn, (HarperCollins College Publishers, NY, USA, 1993).

58. Montalti, M., Credi, A., Prodi, L.,\& Gandofi, M. T., Handbook of Photochemistry 3rd edn (CRC Press, Boca Raton, FL, USA 2006). 